TRANSACTIONS OF THE

AMERICAN MATHEMATICAL SOCIETY

Volume 352, Number 12, Pages 5795-5815

S 0002-9947(00)01959-0

Article electronically published on August 3, 2000

\title{
THE NATURAL REPRESENTATION OF THE STABILIZER OF FOUR SUBSPACES
}

\author{
JOZSEF HORVATH AND ROGER HOWE
}

\begin{abstract}
Consider the natural action of the general linear group $G L(V)$ on the product of four Grassmann varieties of the vector space $V$. In General linear group action on four Grassmannians we gave an algorithm to construct the generic stabilizer $H$ of this action. In this paper we investigate the structure of $V$ as an $H$-module, and we show the effectiveness of the methods developed there, by applying them to the explicit description of $H$.
\end{abstract}

\section{INTRODUCTION}

The study of quadruples of subspaces of a finite dimensional vector space under the action of the general linear group goes back to Nazarova [4] and Gelfand and Ponomarev [1]. It is an example of a "tame problem" of linear algebra; see Kac [3] for an explanation of the terminology. In [2] we developed a method of "successive reductions" to determine the generic stabilizer $H$ of the action of $G L(V)$ on the product $\mathcal{V}=\mathcal{G}(a) \times \mathcal{G}(b) \times \mathcal{G}(c) \times \mathcal{G}(d)$ of four Grassmannians of $V$, where $V$ is a finite dimensional vector space over $\mathbb{C}, \operatorname{dim} V=n$. The goal of this paper is to illustrate the effectiveness of our method, by using it to obtain a complete and concrete description of the group $H$ and its natural representation in $V$. Such explicit descriptions (and the methods by which they are obtained) may also be useful for understanding actions of $G L(V)$ on products of more general flag varieties, including "wild" configurations.

In Section 2 we study the effect of our reductions on the action of the stabilizer. In Section 3 we classify the ways in which the algorithm branches out, depending on the original dimension configuration $(n ; a, b, c, d)$. In Section 4 we determine the structure of the Levi factor $L$ of $H$. In particular we show that $L$ is the product of at most five general linear groups. In Section 5 we give the explicit description of $H$ and its action on $V$. We retain the notation of [2]. Recall that the defect is defined by $\delta=2 n-(a+b+c+d)$, and by duality we assume $\delta \geq 0$.

\section{ACtion AFter REDUCTION}

Theorem 1. Assume that all pairs of $a, b, c, d$ have sum $\leq n$ but all triples have sum $\geq n$. Let

$$
V^{\prime}=(A \oplus B) \cap(C \oplus D), \quad A^{\prime}=A \cap V^{\prime}=A \cap(C \oplus D), \text { etc. }
$$

Received by the editors June 21, 1996.

1991 Mathematics Subject Classification. Primary 20G05; Secondary 14L30, 15A69, 16G20.

Research partially supported by NSF grant DMS-9224358.

(C)2000 American Mathematical Society 
Then the mapping $\alpha$

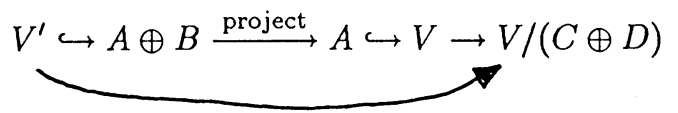

has kernel $A^{\prime} \oplus B^{\prime}$, and the induced map

$$
V^{\prime} /\left(A^{\prime} \oplus B^{\prime}\right) \stackrel{\approx}{\rightarrow} V /(C \oplus D)
$$

is an isomorphism of $\mathrm{H}$-modules. Similarly we obtain an isomorphism of $\mathrm{H}$ modules

$$
V^{\prime} /\left(C^{\prime} \oplus D^{\prime}\right) \stackrel{\approx}{\rightarrow} V /(A \oplus B) .
$$

Proof. Let $v^{\prime} \in \operatorname{ker} \alpha$ and write $v^{\prime}=x+y$ with $x \in A, y \in B$. Then $\alpha\left(v^{\prime}\right)=\bar{x}=\overline{0}$ modulo $(C \oplus D)$, so $x \in(C \oplus D) \cap A=A^{\prime} \subset V^{\prime}$, and $y=v^{\prime}-x \in V^{\prime} \cap B=B^{\prime}$, so $v^{\prime} \in A^{\prime} \oplus B^{\prime}$. Hence ker $\alpha \subset A^{\prime} \oplus B^{\prime}$ and the reverse inclusion is clear. So we have $V^{\prime} /\left(A^{\prime} \oplus B^{\prime}\right) \hookrightarrow V /(C \oplus D)$ and by comparing dimensions,

$$
\begin{aligned}
\operatorname{dim} V^{\prime} /\left(A^{\prime} \oplus B^{\prime}\right) & =n-\delta-(n-\delta-b+n-\delta-a) \\
& =a+b-n+\delta=n-(c+d)=\operatorname{dim} V /(C \oplus D)
\end{aligned}
$$

we see that it is a linear isomorphism. Since all the spaces involved in the definition of $\alpha$ are invariant under $H$, and all the constituent mappings of $\alpha$ are either projections or inclusions, it is clear that $\alpha$ is an $H$-module morphism.

Remark. Proofs similar to that of Theorem 1 will also provide isomorphisms of $H$-modules

$$
\begin{aligned}
& V^{\prime} /\left(A^{\prime} \oplus B^{\prime}\right) \approx A / A^{\prime} \approx \\
& \approx B / B^{\prime} \approx
\end{aligned}(A \oplus B) / V^{\prime}
$$

and

$$
\begin{aligned}
V^{\prime} /\left(C^{\prime} \oplus D^{\prime}\right) & \approx C / C^{\prime} \approx(C \oplus D) / V^{\prime} \\
& \approx D / D^{\prime} \approx
\end{aligned}
$$

Corollary 2. We have isomorphisms of $H$-modules

$$
V / V^{\prime} \approx V /(A \oplus B) \oplus V /(C \oplus D) \approx V^{\prime} /\left(C^{\prime} \oplus D^{\prime}\right) \oplus V^{\prime} /\left(A^{\prime} \oplus B^{\prime}\right)
$$

Proof. The first isomorphism is easy by sending $\bar{v} \in V / V^{\prime}$ to $(\bar{v}, \bar{v}) \in V /(A \oplus B) \oplus$ $V /(C \oplus D)$. The second isomorphism is clear from the theorem.

Corollary 3. Let $(n ; a, b, c, d)$ be such that $r$ consecutive standard reductions are possible, leading to the flag

$$
V \supset V^{\prime} \supset V^{\prime \prime} \supset \cdots \supset V^{(r)} .
$$

Then we have isomorphisms of $H$-modules

$$
V / V^{\prime} \approx V^{\prime} / V^{\prime \prime} \approx \cdots \approx V^{(r-1)} / V^{(r)} .
$$


Corollary 4 (assumptions as in Corollary 3). We have an isomorphism of $L$ modules

$$
V \approx V^{(r)} \oplus\left(\oplus^{r} V / V^{\prime}\right) \approx V^{(r)} \oplus\left(\left(V / V^{\prime}\right) \otimes \mathbb{C}^{r}\right)
$$

where $L$ is a Levi-factor of $H$.

Remark. The above theorem clarifies the effect of standard reduction on the action of the stabilizer. The effect of our other types of reduction is clear from Theorems 3 and 5 of $[2$.

\section{Classification by original Dimensions}

For the rest of the paper we will assume that the dimensions have been ordered $a \geq b \geq c \geq d$. Roughly speaking, the final form of the generic stabilizer $H$ will turn out to depend on the various ways in which the initial dimensions and their duals are distributed in intervals of length $\delta$. This controls the branching of the algorithm according to the questions: (i) In the opening segment of the algorithm, how many pairs are there whose sum exceeds $n$ ? If there are three such pairs, is the third one $b+c$ or $a+d$ ? (ii) In the main part of the algorithm, are there an even or an odd number of standard reductions? (iii) At the start of the 'endgame' of the algorithm, is the sum of all four subspaces direct? If not, then how many triples have direct sum?

Lemma 5. Let $a+b \leq n$. Then after $2 q$ standard reductions the dimensionconfiguration is

$$
(n-2 q \delta ; \quad a-q \delta, \quad b-q \delta, \quad c-q \delta, \quad d-q \delta) .
$$

After $2 q-1$ standard reductions, it is

$$
(n-(2 q-1) \delta ; n-d-q \delta, n-c-q \delta, n-b-q \delta, n-a-q \delta) .
$$

Proof. Clear from Theorem 1 of [2].

Lemma 6. Let $a+b \leq n$. Then $d \leq n-a \leq d+\delta$.

Proof. $a+d \leq a+b \leq n$, so $d \leq n-a . b+c \leq b+a \leq n$, so $a+d=2 n-\delta-(b+c) \geq$ $n-\delta$, so $d+\delta \geq n-a$.

Corollary 7. Let $a+b \leq n$ and $\delta>0$. Let $q$ denote the unique integer such that $(q-1) \delta<d \leq q \delta$. Then $(q-1) \delta<n-a \leq(q+1) \delta$.

Lemma 8. Suppose $a+b \leq n, \delta>0$, and let $q$ be as above.

(1) If $(q-1) \delta<n-a \leq q \delta$, then the process of standard reductions (in the main part of the algorithm) ends after $2(q-1)$ steps.

(2) If $q \delta<n-a \leq(q+1) \delta$, then the process ends after $2 q-1$ steps.

Proof. Verification from Lemma 5.

Lemma 9. Under the conditions of Lemma 8, we have the following 10 mutually exclusive possibilities.

$$
\begin{aligned}
\text { (1) } & (q-1) \delta<n-a \leq q \delta, \text { and } \\
i) & n \leq(2 q-1) \delta ; \text { or } \\
i i) & (2 q-1) \delta<n \leq q \delta+d ; \text { or } \\
i i i) & q \delta+d<n \leq q \delta+c ; \text { or } \\
i v) & q \delta+c<n \leq q \delta+b ; \text { or } \\
v) & q \delta+b<n
\end{aligned}
$$$$
\text { (2) } q \delta<n-a \leq(q+1) \delta \text {, and }
$$$$
\text { i) } 2 q \delta \geq n \text {; or }
$$$$
\text { ii) } n>2 q \delta \geq 2 a \text {; or }
$$$$
\text { iii) } a>q \delta \geq b \text {; or }
$$$$
\text { iv) } b>q \delta \geq c \text {; or }
$$$$
\text { v) } c>q \delta \text {. }
$$ 
Proof. It is clear that case (1) is a disjoint union of i) through v). In case (2) notice that if $2 q \delta \geq n$ then $q \delta>2 q \delta-(n-a) \geq a$, hence i) through v) are disjoint (even if $n<2 a)$.

Corollary 10. With conditions as in Lemma 8, consider the configuration obtained at the end of the process of standard reductions. Then the cases of Lemma 9 correspond to the following outcomes:

(i) the sum of the four subspaces is direct; or

(ii) the sum of the four subspaces is not direct, but all four triples have direct sum; or

(iii) exactly three triples have direct sum; or

(iv) exactly two triples have direct sum; or

(v) exactly one triple has direct sum.

Proof. Direct verification using Lemma 5, Lemma 8 and Lemma 9. As an example, suppose we are in case (1) iii) of Lemma 9 . Then after $2(q-1)$ standard reductions we reach configuration

$$
(n-2(q-1) \delta ; a-(q-1) \delta, b-(q-1) \delta, c-(q-1) \delta, d-(q-1) \delta) .
$$

In this configuration there is exactly one triple whose sum exceeds the dimension of the full space. Namely, we have

$$
(a-(q-1) \delta)+(b-(q-1) \delta)+(c-(q-1) \delta)>n-2(q-1) \delta
$$

but

$$
(a-(q-1) \delta)+(b-(q-1) \delta)+(d-(q-1) \delta) \leq n-2(q-1) \delta,
$$

since the first inequality is equivalent to $n>q \delta+d$ and the second to $n \leq q \delta+c$. The proofs for the other cases are similar.

The above discussion determines the alternative possibilities at the end of the reduction process described in [2]. The next proposition describes the alternatives at the start of the process.

Proposition 11. Let $\tilde{\mathcal{V}}$ be the variety produced by the opening stage of the algorithm (that is, by the removal of intersecting pairs, as in Theorem 5 of [2]). There are 5 mutually exclusive possibilities, depending on the initial configuration $(n ; a, b, c, d)$ as follows:

1. All pairs have sum $\leq n$. Then, obviously, $\tilde{\mathcal{V}}=\mathcal{V}$.

2. There is one pair with sum $>n$, namely $a+b>n$. Then $\tilde{\mathcal{V}}$ has dimension-type $(2 n-a-b ; n-b, n-a, c, d)$.

3. There are two pairs with sum $>n$, namely $a+b$ and $a+c>n$. Then $\tilde{\mathcal{V}}$ has dimension-type

$$
(n-a+d+\delta ; d+\delta, n-a, n-a, d) .
$$

4. There are three pairs with sum $>n$.

i) If $a+b, a+c$ and $a+d>n$, then $\tilde{\mathcal{V}}$ has dimension-type

$$
(2(n-a)+\delta, n-a+\delta, n-a, n-a, n-a) .
$$

ii) If $a+b, a+c$ and $b+c>n$, then $\tilde{\mathcal{V}}$ has dimension-type

$$
(2(d+\delta) ; d+\delta, d+\delta, d+\delta, d)
$$

Proof. Verification using Theorem 5 of [2], once, twice, or three times. 
TABLE 1.

\begin{tabular}{|c|c|}
\hline pairs with sum $>n$ & structure of $H$ \\
\hline none & $\left(\mathbb{C}^{*}\right)^{n / 2}$ \\
$a+b$ & $G L_{a+b-n} \cdot\left(\mathbb{C}^{*}\right)^{d}$ \\
$a+b, a+c$ & $G L_{a+b-n} \cdot G L_{a+c-n} \cdot\left(\mathbb{C}^{*}\right)^{d}$ \\
$a+b, a+c, a+d$ & $G L_{a+b-n} \cdot G L_{a+c-n} \cdot G L_{a+d-n} \cdot\left(\mathbb{C}^{*}\right)^{n-a}$ \\
$a+b, a+c, b+c$ & $G L_{a+b-n} \cdot G L_{a+c-n} \cdot G L_{b+c-n} \cdot\left(\mathbb{C}^{*}\right)^{d}$ \\
\hline
\end{tabular}

\section{LEVI FACTOR}

Proposition 12. Let $\tilde{\mathcal{V}}$ be the variety produced by the opening stage of the algorithm, that is by the removal of all pairs $x, y$ whose sum $x+y>n$. Then we have

$$
H \approx\left(\prod_{x+y>n} G L(x+y-n) \operatorname{Hom}(\delta, x+y-n)\right) \tilde{H} .
$$

Proof. This follows readily from Theorem 5 and the Remark after it in Section 4 of [2].

Corollary 13. If $\delta=0$ then the generic stabilizer $H$ is reductive, and its explicit structure is given in Table 1 . In the natural representation of $H$ all the $G L_{x+y-n}$ components act with multiplicity 1 , and the torus acts with multiplicity 2.

Proof. Clear from Proposition 12, Proposition 11, and Theorem 8 of [2].

Note. Since the above result completely describes the case of defect zero, from now on we will always assume $\delta>0$.

Theorem 14. Let $\delta>0$ and let $H=L U$ be a Levi-decomposition of the generic stabilizer $H$. Then $L$ is the direct product of at most five general linear groups. We describe the explicit structure of $L$ in Table 2. There are 20 mutually exclusive cases. We denote $\tilde{d}=\min \{d, n-a\}$ and $q$ is the unique integer such that $(q-1) \delta<\tilde{d} \leq q \delta$. We have $\tilde{d}=d$ in all cases except case (2) of the table.

Proof. The proof is by carrying out the algorithm presented in [2, according to the classification given in Section 3 above. The list of possibilities arises by combining the 5 possibilities for the initial stage of reduction with the 10 possibilities for the final stage. Since some initial configurations preclude some final configurations, we end with a list of only 20, rather than 50 . For the opening stage of the algorithm (removal of intersecting pairs) we use Propositions 11 and 12, for the stage of standard reductions we use Lemma 8, and for the final stage of the algorithm we use Lemma 9 and Corollary 10. The generic stabilizers of the final configurations are given in Lemma 9 and Theorem 11 of [2]. The details are omitted.

Theorem 15. (Same assumptions as in Theorem 14.) The number of components of $L$ is less than 5 if and only if the condition stated in Table 3 holds. 
TABle 2.

\begin{tabular}{|c|c|c|}
\hline $\begin{array}{l}\text { pairs with } \\
\text { sum }>n\end{array}$ & $\begin{array}{c}\text { further } \\
\text { conditions }\end{array}$ & structure of $L$ \\
\hline (1) $a+b, a+c, b+c$ & none & $G L_{a+b-n} \cdot G L_{a+c-n} \cdot G L_{b+c-n} \cdot G L_{a \delta-d} \cdot G L_{d-(a-1) \delta}$ \\
\hline (2) $a+b, a+c, a+d$ & none & $\begin{array}{l}G L_{a+b-n} \cdot G L_{a+c-n} \cdot G L_{a+d-n} \cdot G L_{q \delta-(n-a)} \cdot G L_{(n-a)-(q-1) \delta} \\
\text { (here } \tilde{d}=n-a)\end{array}$ \\
\hline (3) $a+b, a+c$ & $n-a \leq q \delta$ & $G L_{a+b-n} \cdot G L_{a+c-n} \cdot G L_{n-a-d} \cdot G L_{q \delta-(n-a)} \cdot G L_{d-(q-1) \delta}$ \\
\hline (4) $a+b, a+c$ & $n-a>q \delta$ & $G L_{a+b-n} \cdot G L_{a+c-n} \cdot G L_{q \delta-d} \cdot G L_{n-b-c} \cdot G L_{n-a-q \delta}$ \\
\hline$(5) a+b$ & $n-b \leq q \delta$ & $G L_{a+b-n} \cdot G L_{q \delta-(n-a)} \cdot G L_{c-(q-1) \delta} \cdot G L_{d-(q-1) \delta} \cdot G L_{q \delta-(n-b)}$ \\
\hline (6) $a+b$ & $n-a \leq q \delta<n-b$ & $G L_{a+b-n} \cdot G L_{q \delta-(n-a)} \cdot G L_{n-a-d} \cdot G L_{n-b-q \delta} \cdot G L_{n-a-c}$ \\
\hline (7) $a+b$ & $c \leq q \delta<n-a$ & $G L_{a+b-n} \cdot G L_{q \delta-d} \cdot G L_{n-b-q \delta} \cdot G L_{n-a-q \delta} \cdot G L_{q \delta-c}$ \\
\hline (8) $a+b$ & $q \delta<c$ & $G L_{a+b-n} \cdot G L_{q \delta-d} \cdot G L_{n-b-c} \cdot G L_{c-q \delta} \cdot G L_{n-a-c}$ \\
\hline (9) none & $n \leq(2 q-1) \delta$ & $G L_{a-(q-1) \delta} \cdot G L_{b-(q-1) \delta} \cdot G L_{c-(q-1) \delta} \cdot G L_{d-(q-1) \delta} \cdot G L_{(2 q-1) \delta-n}$ \\
\hline (10) none & $(2 q-1) \delta<n \leq q \delta+d$ & $G L_{q \delta-(n-a)} \cdot G L_{q \delta-(n-b)} \cdot G L_{q \delta-(n-c)} \cdot G L_{q \delta-(n-d)} \cdot G L_{n-(2 q-1) \delta}$ \\
\hline (11) none & $q \delta+d<n \leq q \delta+c$ & $G L_{q \delta-(n-a)} \cdot G L_{q \delta-(n-b)} \cdot G L_{q \delta-(n-c)} \cdot G L_{n-d-q \delta} \cdot G L_{d-(q-1) \delta}$ \\
\hline (12) none & $q \delta+c<n \leq q \delta+b$ & $G L_{q \delta-(n-a)} \cdot G L_{q \delta-(n-b)} \cdot G L_{n-d-q \delta} \cdot G L_{n-c-q \delta} \cdot G L_{n-a-b}$ \\
\hline (13) none & $q \delta+b<n \leq q \delta+a \leq 2 q \delta$ & $G L_{q \delta-(n-a)} \cdot G L_{n-d-q \delta} \cdot G L_{n-c-q \delta} \cdot G L_{n-b-q \delta} \cdot G L_{q \delta-a}$ \\
\hline (14) none & $q \delta+b<n \leq q \delta+a>2 q \delta$ & $G L_{q \delta-(n-a)} \cdot G L_{a-q \delta} \cdot G L_{n-a-b} \cdot G L_{n-a-c} \cdot G L_{n-a-d}$ \\
\hline (15) none & $q \delta+a<n \leq 2 q \delta$ & $G L_{n-d-q \delta} \cdot G L_{n-c-q \delta} \cdot G L_{n-b-q \delta} \cdot G L_{n-a-q \delta} \cdot G L_{2 q \delta-n}$ \\
\hline (16) none & $n>2 q \delta \geq 2 a$ & $G L_{q \delta-d} \cdot G L_{q \delta-c} \cdot G L_{q \delta-b} \cdot G L_{q \delta-a} \cdot G L_{n-2 q \delta}$ \\
\hline (17) none & $\begin{array}{l}q \delta+a<n \\
a>q \delta \geq b\end{array}$ & $G L_{q \delta-d} \cdot G L_{q \delta-c} \cdot G L_{q \delta-b} \cdot G L_{a-q \delta} \cdot G L_{n-a-q \delta}$ \\
\hline (18) none & $b>q \delta \geq c$ & $G L_{q \delta-d} \cdot G L_{q \delta-c} \cdot G L_{a-q \delta} \cdot G L_{b-q \delta} \cdot G L_{n-a-b}$ \\
\hline (19) none & $\begin{array}{l}c>q \delta \\
n-d \leq(q+1) \delta\end{array}$ & $G L_{q \delta-d} \cdot G L_{a-q \delta} \cdot G L_{b-q \delta} \cdot G L_{c-q \delta} \cdot G L_{(q+1) \delta-(n-d)}$ \\
\hline (20) none & $\begin{array}{l}c>q \delta \\
n-d>(q+1) \delta\end{array}$ & $G L_{q \delta-d} \cdot G L_{n-d-(q+1) \delta} \cdot G L_{n-a-b} \cdot G L_{n-a-c} \cdot G L_{n-b}$ \\
\hline
\end{tabular}




\section{TABle 3.}

\begin{tabular}{|c|c|c|c|}
\hline Case & \multicolumn{3}{|c|}{ Condition equivalent to $<5$ components } \\
\hline$(1)$ & $d=q \delta$ & & \\
\hline$(2)$ & $n-a=q \delta$ & & \\
\hline$(3)$ & $n-a=q \delta$ & or & $n=a+d$ \\
\hline$(4)$ & $d=q \delta$ & or & $n=b+c$ \\
\hline$(5)$ & $n-b=q \delta$ & & \\
\hline (6) & $n-a=q \delta$ & or & $n=a+c$ \\
\hline (7) & $c=q \delta$ & & \\
\hline$(8)$ & $d=q \delta$ & or & $n=a+c$ \\
\hline$(9)$ & $n=(2 q-1) \delta$ & & \\
\hline$(10)$ & $n-d=q \delta$ & & \\
\hline$(11)$ & $n-c=q \delta$ & & \\
\hline$(12)$ & $n-b=q \delta$ & or & $n=a+b$ \\
\hline$(13)$ & $n-a=q \delta$ & or & $a=q \delta$ \\
\hline$(14)$ & $n-a=q \delta$ & or & $n=a+b$ \\
\hline$(15)$ & $n=2 q \delta$ & & \\
\hline$(16)$ & $a=q \delta$ & & \\
\hline$(17)$ & $b=q \delta$ & & \\
\hline$(18)$ & $c=q \delta$ & or & $n=a+b$ \\
\hline$(19)$ & $d=q \delta$ & or & $n-d=(q+1) \delta$ \\
\hline$(20)$ & $d=q \delta$ & or & $n=a+b$ \\
\hline
\end{tabular}

Proof. The result follows from examining each of the 20 cases of Theorem 14 . In any given case, if the number of components of $L$ is less than 5 , then at least one of the $G L$ 's must have rank 0 . We can always tell which of the components may have rank 0 . This gives a set of equalities, some of which can be dropped because of dependency between the equalities in the case under consideration. The remaining equalities are the ones listed in the appropriate line of the table. Conversely, it is clear that if any of the equalities listed in the appropriate line of the table holds, then at least one of the components disappears.

As an example, consider Case (7) of Theorem 14. Suppose $L$ has fewer than 5 components. Since $a+b-n>0$, and $q \delta<n-a \leq n-b$, the only $G L$ 's that may have rank 0 are $G L_{q \delta-d}$ and $G L_{q \delta-c}$. Therefore we have $d=q \delta$ or $c=q \delta$. Since $d \leq c \leq q \delta$, we must have $c=q \delta$ (because $d=q \delta$ implies this too). Conversely $c=q \delta$ obviously implies $<5$ components. The other cases are proved similarly.

Corollary 16. (Assumptions as in Theorem 14.) If L has fewer than five components, then either the sum of a pair of $a, b, c, d$ is exactly $n$, or $\delta$ divides one of the numbers $n, a, b, c, d, n-a, n-b, n-c, n-d$.

Proof. By inspection of Table 3.

Corollary 17. (Assumptions as in Theorem 14.) If the sum of a pair of $a, b, c, d$ is exactly $n$, then $L$ has fewer than five components.

Proof. It is left to the reader.

Remark. More informally we might say that $L$ has less than five components if and only if one of the inequalities defining the case to which $L$ belongs is an equality. 
TABLE 4.

\begin{tabular}{|c|c|ccccccc|}
\hline Case & $\begin{array}{c}\text { Defining } \\
\text { condition }\end{array}$ & \multicolumn{5}{c|}{$\begin{array}{c}\text { Structure of } L \text {, including } \\
\text { multiplicities in the action on } V\end{array}$} \\
\hline A1 & $b+c+d=n$ & $G L_{a-d}$ & $\cdot$ & $G L_{a-c}$ & $\cdot$ & $G L_{a-b}$ & $\cdot$ & $G L_{n-2 a}$ \\
& $a \leq n / 2$ & 2 & & 2 & & 2 & & 3 \\
& & & & & & & \\
A2 & $b+c+d=n$ & $G L_{2 a-n}$ & $\cdot$ & $G L_{n-a-d}$ & $\cdot$ & $G L_{n-a-c}$ & $\cdot$ & $G L_{n-a-b}$ \\
& $a>n / 2$ & 3 & & 2 & & 2 & & 2 \\
B & $a+c+d=n$ & $G L_{b-d}$ & $\cdot$ & $G L_{b-c}$ & $\cdot$ & $G L_{c+d-b}$ & $\cdot$ & $G L_{a-b}$ \\
& & 2 & & 2 & & 3 & & 1 \\
C & $a+b+d=n$ & $G L_{c-d}$ & $\cdot$ & $G L_{d}$ & $\cdot$ & $G L_{b-c}$ & $\cdot$ & $G L_{a-c}$ \\
& & 2 & & 3 & & 1 & & 1 \\
D & $a+b+c=n$ & $G L_{d}$ & $\cdot$ & $G L_{c-d}$ & $\cdot$ & $G L_{b-d}$ & $\cdot$ & $G L_{a-d}$ \\
& & 3 & & 1 & & 1 & & 1 \\
\hline
\end{tabular}

Proposition 18. Assume $\delta>0, a+b \leq n$, and there is a triple whose sum is exactly $n$. Let $H=L U$ be a Levi-decomposition of the generic stabilizer $H$. Table 4 gives the structure of $L$, together with the multiplicities of its components in the action on $V$.

Proof. The proof is an extension of the proof of Theorem 11 of [2. The multiplicities follow from Theorem 1 above (and the Remark after Theorem 1). The details are omitted.

\section{EXPLICIT DESCRIPTION}

Theorem 19. Let $\delta>0$, and let $H=L U$ be a Levi-decomposition of the generic stabilizer $H$. Let $\tilde{d}=\min \{d, n-a\}$ and $q$ be the unique integer such that $(q-1) \delta<$ $\tilde{d} \leq q \delta$. Below we give the explicit structure of $H$, in all twenty cases, including the multiplicities of the components of $L$ in the action on $V$, and the structure of the unipotent radical $U$.

Proof. All these results follow from carrying out the algorithm of [2, similarly to the proof of Theorem 14. The $L$-module structure is obtained from Theorem 1 and the Remark after it, and Proposition 18.

Case (1). $a+b, a+c, b+c>n$.

$(n ; a, b, c, d)$

( 3 reductions by Theorem 5 of 2 ]

$(2 d+2 \delta ; d+\delta, d+\delta, d+\delta, d)$

( $2 q-1$ reductions by Theorem 1 of [2]

$(2 d-(2 q-3) \delta ; d-(q-2) \delta, d-(q-1) \delta, d-(q-1) \delta, d-(q-1) \delta)$

( reduction by Theorem 3 of 2 ]

$(3 d-3(q-1) \delta ; 2 d-2(q-1) \delta, d-(q-1) \delta, d-(q-1) \delta, d-(q-1) \delta)$

Case A2 of Proposition 18. 


\section{Structure of $H$.}

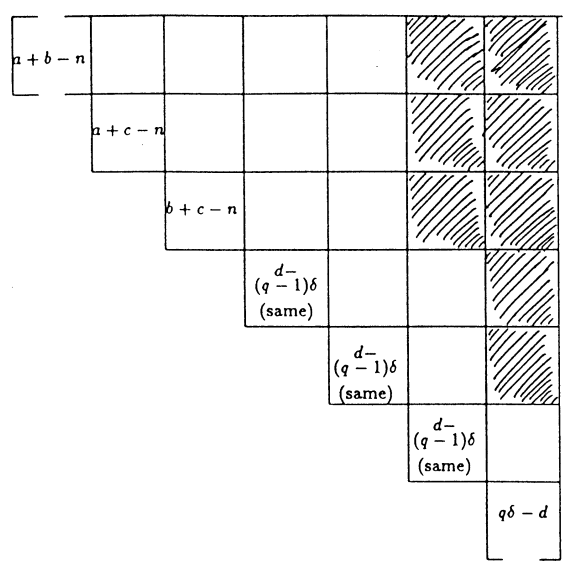

\section{$L$-module-structure.}

$$
V=\mathbb{C}^{a+b-n} \oplus \mathbb{C}^{a+c-n} \oplus \mathbb{C}^{b+c-n} \oplus(2 q+2) \mathbb{C}^{d-(q-1) \delta} \oplus 2 q \mathbb{C}^{q \delta-d}
$$

Case (2). $a+b, a+c, a+d>n \quad(\tilde{d}=n-a)$

$(n ; a, b, c, d)$

( 3 reductions by Theorem 5 of 2

$(2(n-a)+\delta ; n-a+\delta, n-a, n-a, n-a)$

( $2(q-1)$ reductions by Theorem 1 of 2

$(2(n-a)-(2 q-3) \delta ; n-a-(q-2) \delta, n-a-(q-1) \delta$

( reduction by Theorem 3 of [2]

$(3(n-a)-3(q-1) \delta ; 2(n-a)-2(q-1) \delta$,

$n-a-(q-1) \delta, n-a-(q-1) \delta, n-a-(q-1) \delta)$

Case A2 of Proposition 18

\section{Structure of $H$.}

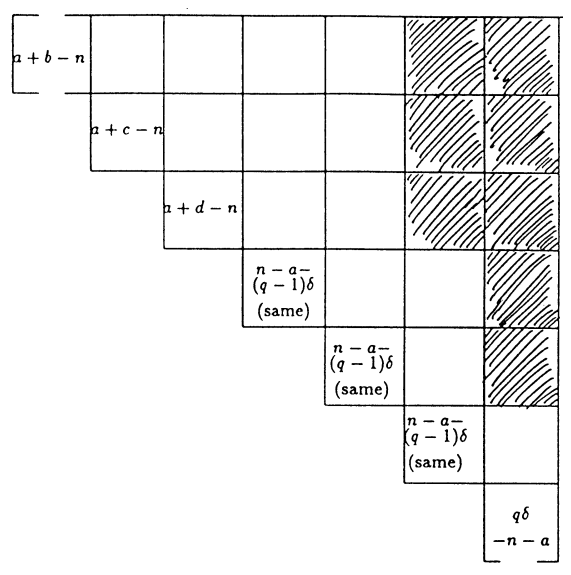




\section{$L$-module structure.}

$$
V=\mathbb{C}^{a+b-n} \oplus \mathbb{C}^{a+c-n} \oplus \mathbb{C}^{a+d-n} \oplus(2 q+1) \mathbb{C}^{n-a-(q-1) \delta} \oplus(2 q-1) \mathbb{C}^{q \delta-n+a}
$$

Case (3). $a+b, a+c>n$, all other pairs $\leq n, n-a \leq q \delta$

$(n ; a, b, c, d)$

( 2 reductions by Theorem 5 of 2 ]

$(n-a+d+\delta ; d+\delta, n-a, n-a, d)$

( $2(q-1)$ reductions by Theorem 1 of [2]

$(n-a+d-(2 q-3) \delta ; d-(q-2) \delta, n-a-(q-1) \delta, n-a-(q-1) \delta, d-(q-1) \delta)$

( reduction by Theorem 3 of [2]

$(2(n-a)+d-3(q-1) \delta ; n-a+d-2(q-1) \delta$,

$n-a-(q-1) \delta, n-a-(q-1) \delta, d-(q-1) \delta)$

Case A2 of Proposition 18

\section{Structure of $H$.}

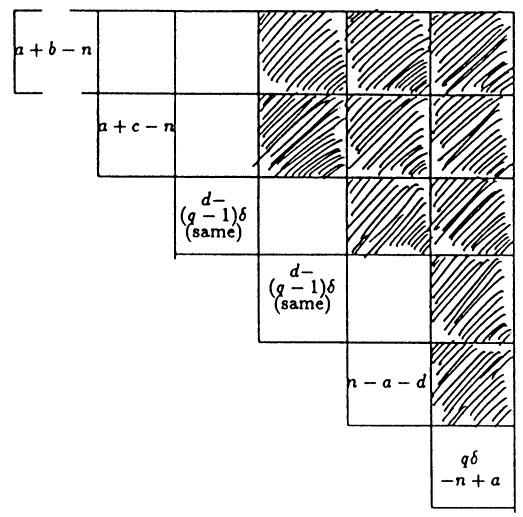

\section{$L$-module structure.}

$$
V=\mathbb{C}^{a+b-n} \oplus \mathbb{C}^{a+c-n} \oplus(2 q+1) \mathbb{C}^{d-(q-1) \delta} \oplus 2 q \mathbb{C}^{n-a-d} \oplus(2 q-1) \mathbb{C}^{q \delta-n+a}
$$

Case (4). $a+b, a+c>n$, all other pairs $\leq n, n-a>q \delta$.

$(n ; a, b, c, d)$

( 2 reductions by Theorem 5 of 2 ]

$(n-a+d+\delta ; d+\delta, n-a, n-a, d)$

$(2 q-1$ reductions by Theorem 1 of [2]

$(n-a+d-2(q-1) \delta ; n-a-(q-1) \delta, d-(q-1) \delta, d-(q-1) \delta, n-a-q \delta)$

( reduction by Theorem 3 of [2]

$(n-a+2 d-(3 q-2) \delta ; n-a+d-(2 q-1) \delta, d-(q-1) \delta, d-(q-1) \delta, n-a-q \delta)$

Case A 2 of Proposition 18 


\section{Structure of $H$.}

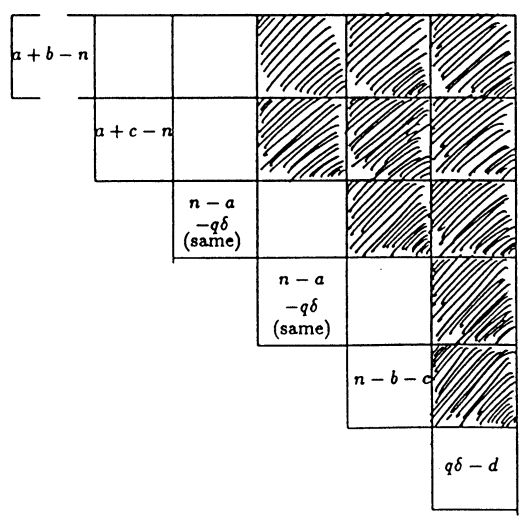

\section{$L$-module structure.}

$$
V=\mathbb{C}^{a+b-n} \oplus \mathbb{C}^{a+c-n} \oplus(2 q+2) \mathbb{C}^{n-a-q \delta} \oplus(2 q+1) \mathbb{C}^{n-b-c} \oplus 2 q \mathbb{C}^{q \delta-d}
$$

Case (5). $a+b>n$, all other pairs $\leq n, n-b \leq q \delta$.

$(n ; a, b, c, d)$

( reduction by Theorem 5 of 2 ]

$(2 n-a-b ; n-b, n-a, c, d)$

$(2(q-1)$ reductions by Theorem 1 of [2]

$(2 n-a-b-2(q-1) \delta ; n-b-(q-1) \delta, n-a-(q-1) \delta, c-(q-1) \delta, d-(q-1) \delta)$ ( reduction by Theorem 3 of [2]

$(n-a+c+d-3(q-1) \delta ; n-a-(q-1) \delta, c+d-2(q-1) \delta, c-(q-1) \delta$, $d-(q-1) \delta)$

Case B of Proposition 18

\section{Structure of $H$.}

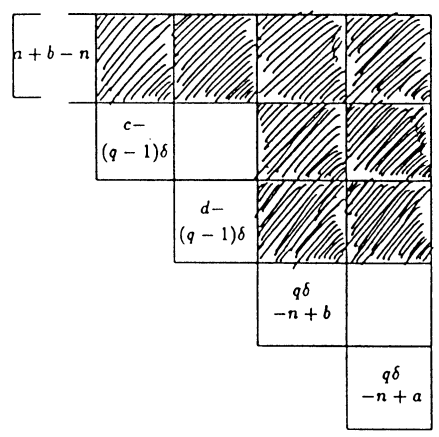

\section{$L$-module structure.}

$$
V=\mathbb{C}^{a+b-n} \oplus 2 q \mathbb{C}^{c-(q-1) \delta} \oplus 2 q \mathbb{C}^{d-(q-1) \delta} \oplus(2 q-1) \mathbb{C}^{q \delta-n+b} \oplus(2 q-1) \mathbb{C}^{q \delta-n+a}
$$


Case (6). $a+b>n$, all other pairs $\leq n, n-a \leq q \delta<n-b$.

$(n ; a, b, c, d)$

( reduction by Theorem 5 of [2]

( $(2 n-a-b ; n-b, n-a, c, d)$

$(2(q-1)$ reductions by Theorem 1 of [2]

$(2 n-a-b-2(q-1) \delta ; n-b-(q-1) \delta, n-a-(q-1) \delta, c-(q-1) \delta, d-(q-1) \delta)$

( reduction by Theorem 3 of [2]

$(n-a+c+d-3(q-1) \delta ; c+d-2(q-1) \delta, n-a-(q-1) \delta$,

$c-(q-1) \delta, d-(q-1) \delta)$

Case A2 of Proposition 18

\section{Structure of $H$.}

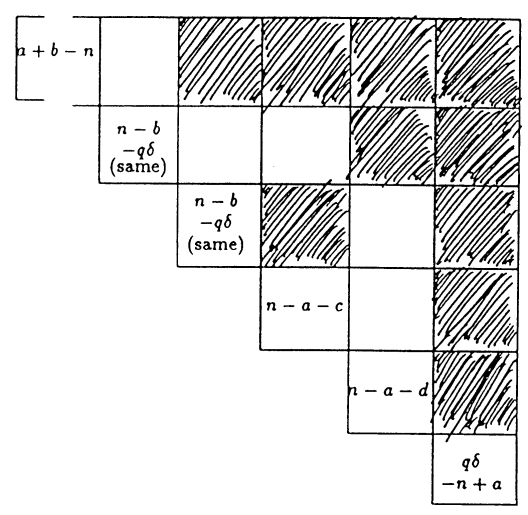

\section{$L$-module structure.}

$$
V=\mathbb{C}^{a+b-n} \oplus(2 q+1) \mathbb{C}^{n-b-q \delta} \oplus 2 q \mathbb{C}^{n-a-c} \oplus 2 q \mathbb{C}^{n-a-d} \oplus(2 q-1) \mathbb{C}^{q \delta-n+a}
$$

Case (7). $a+b>n$, all other pairs $\leq n, c \leq q \delta<n-a$.

$(n ; a, b, c, d)$

( reduction by Theorem 5 of 2 .

$(2 n-a-b ; n-b, n-a, c, d)$

( $2 q-1$ reductions by Theorem 1 of 2 ]

$(c+d-2(q-1) \delta ; c-(q-1) \delta, d-(q-1) \delta, n-b-q \delta, n-a-q \delta)$

( reduction by Theorem 3 of 2 ]

$(2 n-a-b+d-(3 q-1) \delta ; d-(q-1) \delta, 2 n-a-b-2 q \delta, n-b-q \delta, n-a-q \delta)$

Case B of Proposition 18 


\section{Structure of $H$.}

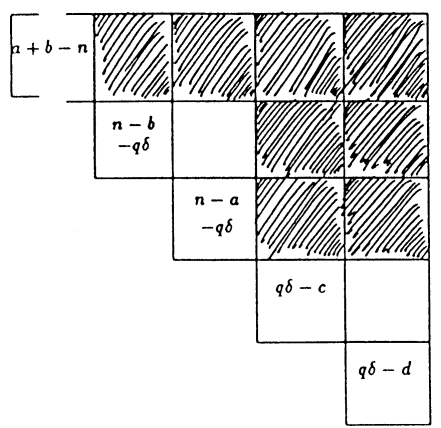

\section{$L$-module structure.}

$$
V=\mathbb{C}^{a+b-n} \oplus(2 q+1) \mathbb{C}^{n-b-q \delta} \oplus(2 q+1) \mathbb{C}^{n-a-q \delta} \oplus 2 q \mathbb{C}^{q \delta-c} \oplus 2 q \mathbb{C}^{q \delta-d}
$$

Case (8). $a+b>n$, all other pairs $\leq n, q \delta<c$.

$(n ; a, b, c, d)$

( reduction by Theorem 5 of [2]

$(2 n-a-b ; n-b, n-a, c, d)$

$((2 q-1)$ reductions by Theorem 1 of $[2]$

$(c+d-2(q-1) \delta ; c-(q-1) \delta, d-(q-1) \delta, n-b-q \delta, n-a-q \delta)$

( reduction by Theorem 3 of [2]

$(2 n-a-b+d-(3 q-1) \delta ; 2 n-a-b-2 q \delta, d-(q-1) \delta, n-b-q \delta, n-a-q \delta)$

Case A2 of Proposition 18

\section{Structure of $H$.}

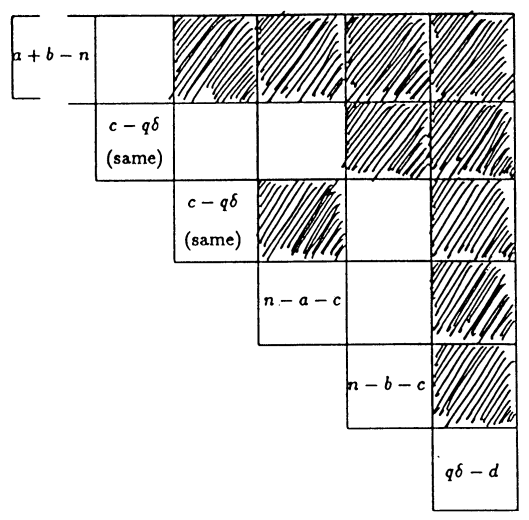

\section{$L$-module structure.}

$$
V=\mathbb{C}^{a+b-n} \oplus(2 q+2) \mathbb{C}^{c-q \delta} \oplus(2 q+1) \mathbb{C}^{n-a-c} \oplus(2 q+1) \mathbb{C}^{n-b-c} \oplus 2 q \mathbb{C}^{q \delta-d}
$$


Case (9). All pairs $\leq n, n \leq(2 q-1) \delta$.

$(n ; a, b, c, d)$

( $2(q-1)$ reductions by Theorem 1 of [2]

$(n-2(q-1) \delta ; a-(q-1) \delta, b-(q-1) \delta, c-(q-1) \delta, d-(q-1) \delta)$ sum of all four spaces is direct

\section{Structure of $H$.}

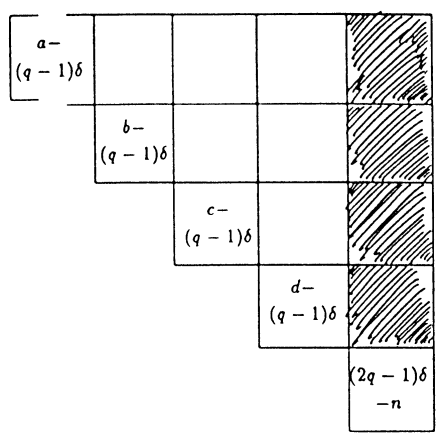

$L$-module structure.

$$
\begin{aligned}
& V=(2 q-1) \mathbb{C}^{a-(q-1) \delta} \oplus(2 q-1) \mathbb{C}^{b-(q-1) \delta} \oplus(2 q-1) \mathbb{C}^{c-(q-1) \delta} \\
& \oplus(2 q-1) \mathbb{C}^{d-(q-1) \delta} \oplus(4 q-3) \mathbb{C}^{(2 q-1) \delta-n}
\end{aligned}
$$

Case (10). All pairs $\leq n, n>(2 q-1) \delta, n-d \leq q \delta$.

$(n ; a, b, c, d)$

( $2(q-1)$ reductions by Theorem 1 of $[2]$

$(n-2(q-1) \delta ; a-(q-1) \delta, b-(q-1) \delta, c-(q-1) \delta, d-(q-1) \delta)$

( reduction by Theorem 3 of 2

$(b+c+d-3(q-1) \delta ; b-(q-1) \delta, c-(q-1) \delta, d-(q-1) \delta, n-(2 q-1) \delta)$

Case D of Proposition 18

\section{Structure of $H$.}

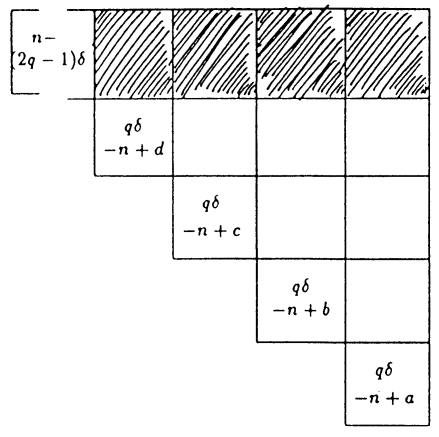

$L$-module structure.

$$
\begin{aligned}
V=(4 q-1) \mathbb{C}^{n-(2 q-1) \delta} & \oplus(2 q-1) \mathbb{C}^{q \delta-n+d} \oplus(2 q-1) \mathbb{C}^{q \delta-n+c} \\
& \oplus(2 q-1) \mathbb{C}^{q \delta-n+b} \oplus(2 q-1) \mathbb{C}^{q \delta-n+a}
\end{aligned}
$$


Case (11). All pairs $\leq n, n-c \leq q \delta<n-d$.

$(n ; a, b, c, d)$

( $2(q-1)$ reductions by Theorem 1 of [2]

$(n-2(q-1) \delta ; a-(q-1) \delta, b-(q-1) \delta, c-(q-1) \delta, d-(q-1) \delta)$

( reduction by Theorem 3 of [2]

$(b+c+d-3(q-1) \delta ; b-(q-1) \delta, c-(q-1) \delta, n-(2 q-1) \delta, d-(q-1) \delta)$

Case $\mathrm{C}$ of Proposition 18

\section{Structure of $H$.}

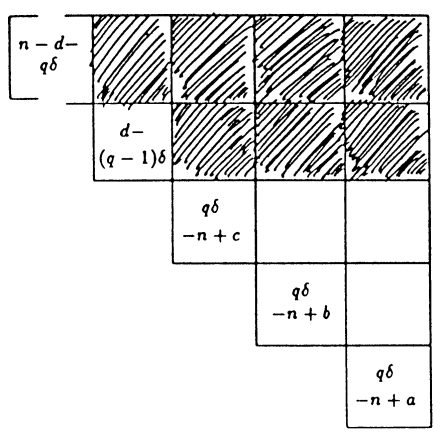

$L$-module structure.

$$
\begin{aligned}
& V=2 q \mathbb{C}^{n-d-q \delta} \oplus(4 q-1) \mathbb{C}^{d-(q-1) \delta} \oplus(2 q-1) \mathbb{C}^{q \delta-n+c} \\
& \oplus(2 q-1) \mathbb{C}^{q \delta-n+b} \oplus(2 q-1) \mathbb{C}^{q \delta-n+a}
\end{aligned}
$$

Case (12). All pairs $\leq n, n-b \leq q \delta<n-c$.

$(n ; a, b, c, d)$

( $2(q-1)$ reductions by Theorem 1 of [2]

$(n-2(q-1) \delta ; a-(q-1) \delta, b-(q-1) \delta, c-(q-1) \delta, d-(q-1) \delta)$

( reduction by Theorem 3 of [2]

$(b+c+d-3(q-1) \delta ; b-(q-1) \delta, n-(2 q-1) \delta, c-(q-1) \delta, d-(q-1) \delta)$

Case B of Proposition 18

\section{Structure of $H$.}

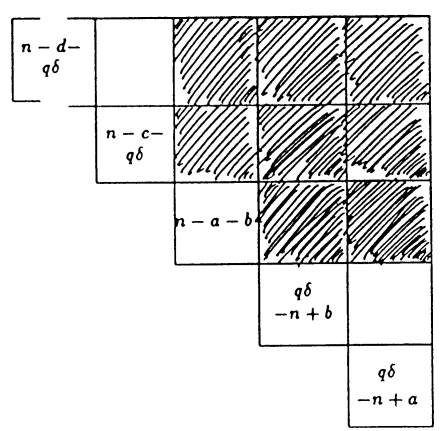




\section{$L$-module structure.}

$$
\begin{aligned}
V=2 q \mathbb{C}^{n-d-q \delta} & \oplus 2 q \mathbb{C}^{n-c-q \delta} \oplus(4 q-1) \mathbb{C}^{n-a-b} \\
& \oplus(2 q-1) \mathbb{C}^{q \delta-n+b} \oplus(2 q-1) \mathbb{C}^{q \delta-n+a} .
\end{aligned}
$$

Case (13). All pairs $\leq n, n-a \leq q \delta<n-b, a \leq q \delta$.

$(n ; a, b, c, d)$

( $2(q-1)$ reductions by Theorem 1 of [2]

$((n-2(q-1) \delta ; a-(q-1) \delta, b-(q-1) \delta, c-(q-1) \delta, d-(q-1) \delta)$

( reduction by Theorem 3 of 2

$(b+c+d-3(q-1) \delta ; n-(2 q-1) \delta, b-(q-1) \delta, c-(q-1) \delta, d-(q-1) \delta)$

Case A1 of Proposition 18

\section{Structure of $H$.}

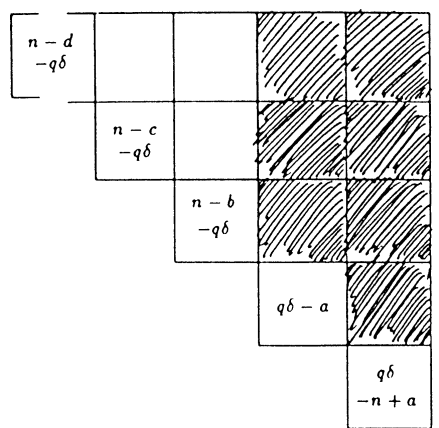

\section{$L$-module structure.}

$$
\begin{aligned}
V=2 q \mathbb{C}^{n-d-q \delta} & \oplus 2 q \mathbb{C}^{n-c-q \delta} \oplus 2 q \mathbb{C}^{n-b-q \delta} \\
& \oplus(4 q-1) \mathbb{C}^{q \delta-a} \oplus(2 q-1) \mathbb{C}^{q \delta-n+a}
\end{aligned}
$$

Case (14). All pairs $\leq n, n-a \leq q \delta<n-b, a>q \delta$

$(n ; a, b, c, d)$

( $2(q-1)$ reductions by Theorem 1 of 2

$(n-2(q-1) \delta ; a-(q-1) \delta, b-(q-1) \delta, c-(q-1) \delta, d-(q-1) \delta)$

( reduction by Theorem 3 of [2]

$(b+c+d-3(q-1) \delta ; n-(2 q-1) \delta, b-(q-1) \delta, c-(q-1) \delta, d-(q-1) \delta)$

Case A2 of Proposition 18 


\section{Structure of $H$.}

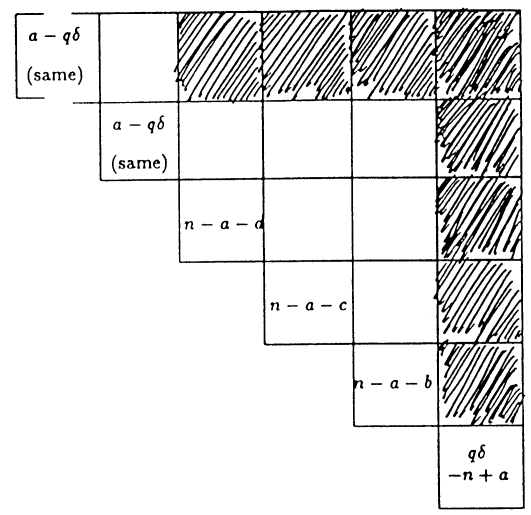

$L$-module structure.

$$
\begin{aligned}
& V=(2 q+1) \mathbb{C}^{a-q \delta} \oplus 2 q \mathbb{C}^{n-a-d} \oplus 2 q \mathbb{C}^{n-a-c} \\
& \oplus 2 q \mathbb{C}^{n-a-b} \oplus(2 q-1) \mathbb{C}^{q \delta-n+a}
\end{aligned}
$$

Case (15). All pairs $\leq n, q \delta<n-a, n \leq 2 q \delta$.

$(n ; a, b, c, d)$

$(2 q-1$ reductions by Theorem 1 of 2

$(n-(2 q-1) \delta ; n-d-q \delta, n-c-q \delta, n-b-q \delta, n-a-q \delta)$ sum of all four spaces is direct

\section{Structure of $H$.}

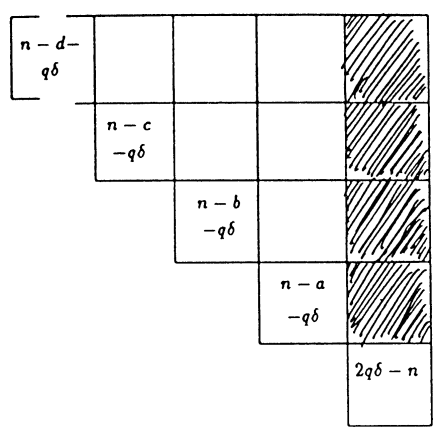

$L$-module structure.

$$
\begin{aligned}
V=2 q \mathbb{C}^{n-d-q \delta} & \oplus 2 q \mathbb{C}^{n-c-q \delta} \oplus 2 q \mathbb{C}^{n-b-q \delta} \\
& \oplus 2 q \mathbb{C}^{n-a-q \delta} \oplus(4 q-1) \mathbb{C}^{2 q \delta-n}
\end{aligned}
$$


Case (16). All pairs $\leq n, q \delta<n-a, n>2 q \delta, a \leq q \delta$.

$(n ; a, b, c, d)$

( $2 q-1$ reductions by Theorem 1 of $[2]$ $(n-(2 q-1) \delta ; n-d-q \delta, n-c-q \delta, n-b-q \delta, n-a-q \delta)$

( reduction by Theorem 3 of [2]

$(3 n-a-b-c-3 q \delta ; n-c-q \delta, n-b-q \delta, n-a-q \delta, n-2 q \delta)$

Case D of Proposition 18

\section{Structure of $H$.}

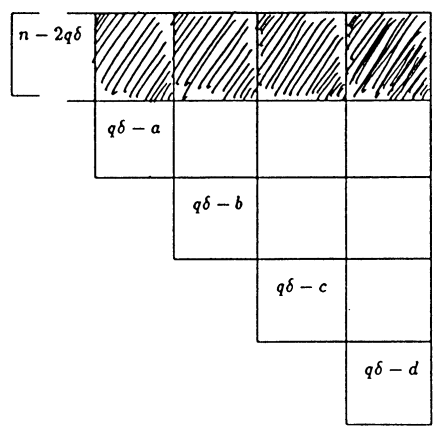

$L$-module structure.

$$
\begin{aligned}
V=(4 q+1) \mathbb{C}^{n-2 q \delta} & \oplus 2 q \mathbb{C}^{q \delta-a} \oplus 2 q \mathbb{C}^{q \delta-b} \\
& \oplus 2 q \mathbb{C}^{q \delta-c} \oplus 2 q \mathbb{C}^{q \delta-d}
\end{aligned}
$$

Case (17). All pairs $\leq n, q \delta<n-a, b \leq q \delta<a$ $(n ; a, b, c, d)$

( $(2 q-1)$ reductions by Theorem 1 of $[2]$ $(n-(2 q-1) \delta ; n-d-q \delta, n-c-q \delta, n-b-q \delta, n-a-q \delta)$

( reduction by Theorem 3 of [2]

$(3 n-a-b-c-3 q \delta ; n-c-q \delta, n-b-q \delta, n-2 q \delta, n-a-q \delta)$ Case $\mathrm{C}$ of Proposition 18

\section{Structure of $H$.}

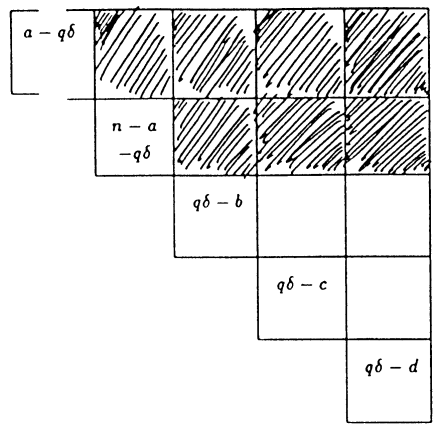


$L$-module structure.

$$
\begin{aligned}
V=(2 q+1) \mathbb{C}^{a-q \delta} & \oplus(4 q+1) \mathbb{C}^{n-a-q \delta} \oplus 2 q \mathbb{C}^{q \delta-b} \\
& \oplus 2 q \mathbb{C}^{q \delta-c} \oplus 2 q \mathbb{C}^{q \delta-d}
\end{aligned}
$$

Case (18). All pairs $\leq n, c \leq q \delta<b$.

$(n ; a, b, c, d)$

( $2 q-1$ reductions by Theorem 1 of [2]

$(n-(2 q-1) \delta ; n-d-q \delta, n-c-q \delta, n-b-q \delta, n-a-q \delta)$

( reduction by Theorem 3 of 2 ]

$(3 n-a-b-c-3 q \delta ; n-c-q \delta, n-2 q \delta, n-b-q \delta, n-a-q \delta)$

Case B of Proposition 18

\section{Structure of $H$.}

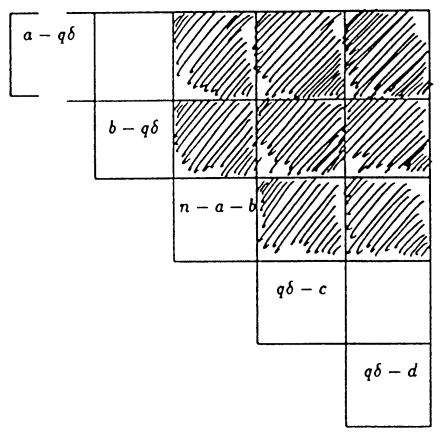

\section{$L$-module structure.}

$$
\begin{aligned}
V=(2 q+1) \mathbb{C}^{a-q \delta} & \oplus(2 q+1) \mathbb{C}^{b-q \delta} \oplus(4 q+1) \mathbb{C}^{n-a-b} \\
& \oplus 2 q \mathbb{C}^{q \delta-c} \oplus 2 q \mathbb{C}^{q \delta-d}
\end{aligned}
$$

Case (19). All pairs $\leq n, q \delta<c, n-d \leq(q+1) \delta$

$(n ; a, b, c, d)$

( $2 q-1$ reductions by Theorem 1 of $[2]$

$(n-(2 q-1) \delta ; n-d-q \delta, n-c-q \delta, n-b-q \delta, n-a-q \delta)$

( reduction by Theorem 3 of 2

$(3 n-a-b-c-3 q \delta ; n-2 q \delta, n-c-q \delta, n-b-q \delta, n-a-q \delta)$

Case A1 of Proposition 18 


\section{Structure of $H$.}

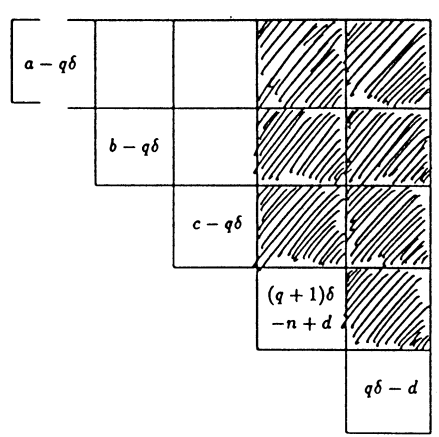

$L$-module structure.

$$
\begin{aligned}
V=(2 q+1) \mathbb{C}^{a-q \delta} \oplus(2 q+1) \mathbb{C}^{b-q \delta} \oplus(2 q+1) \mathbb{C}^{c-q \delta} \\
\oplus(4 q+1) \mathbb{C}^{(q+1) \delta-n+d} \oplus 2 q \mathbb{C}^{q \delta-d}
\end{aligned}
$$

Case (20). All pairs $\leq n, q \delta<c, n-d>(q+1) \delta$

$(n ; a, b, c, d)$

( $2 q-1$ reductions by Theorem 1 of [2]

$(n-(2 q-1) \delta ; n-d-q \delta, n-c-q \delta, n-b-q \delta, n-a-q \delta)$

( reduction by Theorem 3 of [2]

$(3 n-a-b-c-3 q \delta ; n-2 q \delta, n-c-q \delta, n-b-q \delta, n-a-q \delta)$

Case A2 of Proposition 18

Structure of $H$.

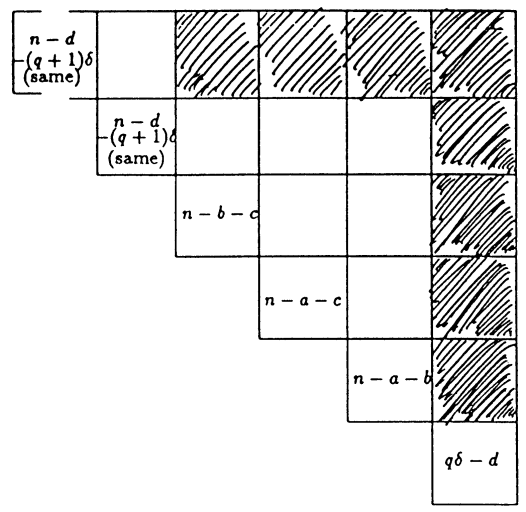

$L$-module structure.

$$
\begin{aligned}
V=(2 q+2) \mathbb{C}^{n-d-(q+1) \delta} & \oplus(2 q+1) \mathbb{C}^{n-b-c} \oplus(2 q+1) \mathbb{C}^{n-a-c} \\
& \oplus(2 q+1) \mathbb{C}^{n-a-b} \oplus 2 q \mathbb{C}^{q \delta-d}
\end{aligned}
$$




\section{REFERENCES}

1. I.M. Gelfand and V.A. Ponomarev, Problems of linear algebra and classification of quadruples of subspaces in a finite dimensional vector space, Coll. Math. Soc. Bolyai, Tihany (Hungary) North-Holland, 1972, pp. 163-237. MR 50:9896

2. J. Horvath and R. Howe, General linear group action on four Grassmannians, submitted to Mathematische Zeitschrift.

3. V. Kac, Infinite root systems, representations of graphs and invariant theory, Invent. Math. 56 (1980), 57-92. MR 82j:16050

4. L.A. Nazarova, Representations of quadruples, Izv. Akad. Nauk SSSR Ser. Mat. 31 (1967), 1361-1377. MR 36:6400

Department of Mathematics, West Chester University, West Chester, Pennsylvania 19383

Department of Mathematics, Yale University, 10 Hillhouse Avenue, New Haven, ConNECTICUT 06520-8283 